\title{
INDUSTRY WASTEWATER POLLUTION CONTROL BY ADSORPTION-A LOW COST PROMISING PHYSICO-CHEMICAL TREATMENT
}

\section{HEMAXI KAPURIYA ${ }^{1}$, DR. HITESH DESAI ${ }^{2} \& *$ DR. HEMANGI DESAI $^{3}$}

${ }^{I}$ TIFAC-CORE in Environmental Engineering, SCET, Surat

${ }^{2}$ Sarvajanik College of Engineering \& Technology, Surat

${ }^{3}$ Shree Ramkrishna Institute of Computer Education \& Applied Sciences, Surat

Received: Oct 11, 2020; Accepted: Oct 31, 2020; Published: Nov 20, 2020; Paper Id.: IJEEFUSOCT20201

\section{INTRODUCTION}

Waste water from manufacturing or chemical processes in industries contributes to water pollution. Industrial waste water usually contains specific and readily identifiable chemical compounds. Water pollution is concentrated within a few subsectors, mainly in the form of toxic wastes and organic pollutants.Large number of chemical, diamond, textile, iron and steel, cement, pharmaceuticals and many other industries are running now-a-days as a result of technical progress. As a result of rapidly expanding industrialization, urbanization and population blast, our 
streams,lakes, ponds and other water bodies are being polluted. Water is regarded as polluted when it is changed in its quality or composition directly or indirectly as a result of human activities. [1]

One of the most devastating effects of industrial waste is water pollution. For most industrial processes, heavy amount of water is used which comes in contact with harmful chemicals. These chemicals are usually metals or radioactive material. This heavily effects the environment because most of waste ends up in oceans, lakes, or rivers. As a result, water becomes polluted posing as health hazard to everyone. Farmers rely on this water but if the water is polluted, then crops that are produced can become polluted. These affect the health of society because if industrial companies can't clean up their waste, this begins to affect the life of humans but also animals. Sea creature's health is affected because their lives become endangered by this polluted water. Water pollution can have devastating effects on the human body with the main ones being infections from bacteria, parasites, and chemicals. Diseases that humans can be exposed from drinking unsafe water range from cholera, typhoid, or Giardia.

Today the water pollution becomes a major problem in the whole world. This method is helpful in treatment of ground water pollution [10] due to industrial effluents and also in direct treatment of industrial effluents.This study will give the alternative approach to the industrial waste water treatment by Guava leaves powder as low cost adsorbent is ecofriendly and natural treatment will save the chemical cost used for treatment. This treatment is non-hazardous to the human health, environment and whole ecosystem. As Guava leaves powder is biodegradable in nature, after treatment it will not create any harmful effects to the environment.

\section{MATERIALS AND METHODS}

\section{COLLECTION OF SAMPLE}

1. Industrial effluents: were collected from Ankleashwar, GIDC.

2. GUAVA leaves: Fresh Guava leaves were harvested from Guava tree available at Ved gam, Surat.

\section{PREPARATION OF ADSORBENT}

The collected material was thoroughly washed with distilled water three to four times to remove dust and other impurities. Then sun dried the washed leaves. Dried leaves were then grinded and sieved. $250 \mu \mathrm{m}$ particle size of GLP obtained after sieving.Dried GLP was kept in plastic containers and stored in dry place. [2]

\section{APPLICATION OF DOSAGE}

Here, in first phase waste water is treated with $10 \mathrm{gm} / \mathrm{GLP}$ at different contact time 30, 60, 90, $120 \mathrm{~min}$ and stirring at 120 rpm and at $\mathrm{pH} 7$.

In second phase waste water was treated at 30 min contact time with 5,10,15,20 gm/l GLP dose and stirring at $120 \mathrm{rpm}$ and at $\mathrm{pH} 7$. 


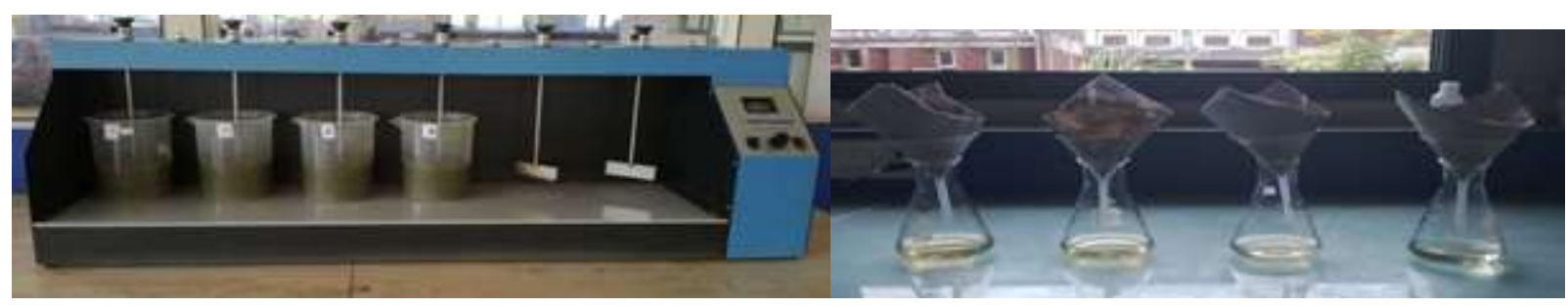

Figure 1: Jar Test apparatus for setting dosage of GLP with effluent sample

\section{METHOD OF ANALYSIS}

Analysis of samples before and after treatment with GLP was carried out by following method given in the table 1. Results of the analysis were compared with the standards mention in following table with the reference of WHO guideline. Methods were followed with the reference of APHA. [3]

Table 1: Standard Methods and Permissible Limits

\begin{tabular}{|c|c|c|c|}
\hline Method & Parameter & $\begin{array}{l}\text { Drinking water } \\
\text { standard }\end{array}$ & $\begin{array}{l}\text { Waste water Surface } \\
\text { Inland standard }\end{array}$ \\
\hline $\mathrm{pH}$ meter & $\mathrm{pH}$ & $6.5-8.5$ & $5.5-9.0$ \\
\hline Turbidity meter & Turbidity & $1 \mathrm{NTU}$ & $10 \mathrm{NTU}$ \\
\hline EDTA titration & Total Hardness & $300 \mathrm{mg} / \mathrm{l}$ & - \\
\hline EDTA titration & Ca Hardness & $75 \mathrm{mg} / \mathrm{l}$ & - \\
\hline EDTA titration & Mg Hardness & $30 \mathrm{mg} / \mathrm{l}$ & - \\
\hline Open refluxing & COD & - & $250 \mathrm{mg} / \mathrm{l}$ \\
\hline Titration & BOD & - & $30 \quad \mathrm{mg} / \mathrm{l}$ \\
\hline Drying and Gravimetric & TDS & $500 \mathrm{mg} / \mathrm{l}$ & $2100 \mathrm{mg} / \mathrm{l}$ \\
\hline Drying and Gravimetric & TSS & - & $100 \mathrm{mg} / \mathrm{l}$ \\
\hline Titration & Iron & $0.3 \mathrm{mg} / 1$ & $3.0 \mathrm{mg} / \mathrm{l}$ \\
\hline Spectrophotometric & Nitrite & - & - \\
\hline Spectrophotometric & Nitrate & $45 \mathrm{mg} / \mathrm{l}$ & $10 \mathrm{mg} / \mathrm{l}$ \\
\hline Spectrophotometric & Chromium Hexavalent & $0.05 \mathrm{mg} / \mathrm{l}$ & $0.1 \mathrm{mg} / \mathrm{l}$ \\
\hline
\end{tabular}

\section{Batch Isotherm Studies}

Isotherm experiments were conducted toinvestigate the relationship between the solidphase concentration of an adsorbate and the solution phase concentration of the adsorbateat an equilibrium condition. The removal percentage (R \%) of SDS was calculated for each run by following equation: $\mathrm{R}(\%)=[(\mathrm{Ci}-\mathrm{Ce}) / \mathrm{Ci}] * 100$, Where, $\mathrm{Ci}$ and $\mathrm{Ce}$ are the initial and final concentration of parameter $(\mathrm{mg} / \mathrm{L})$ in the solution. The adsorption capacity of the adsorbent for each concentration of parameter at equilibrium was calculated using following equation: qe $(\mathrm{mg} / \mathrm{g})=[(\mathrm{Ci}-\mathrm{Ce}) / \mathrm{M}] * \mathrm{~V}$, Where, Ci and Ce were the initial and final concentration of parameter $(\mathrm{mg} / \mathrm{L})$ in the test solution respectively. $\mathrm{V}$ is the volume of solution (in Litter) and $\mathrm{M}$ is the mass of adsorbent (gm). [3,4]

\section{RESULT AND DISCUSSIONS}

\section{Result for Effect Of Different Contact Time on Adsorption of Industrial Waste Water}

Analysis of effluent before treated with Guava leaves adsorbent showed that values of all the water quality parameters were observed above the discharge limit. 
Result of waste water quality parameter after treatment with $10 \mathrm{gm} / 1 \mathrm{GLP}$ at different contact time 30, 60, 90, 120 min and stirring at $120 \mathrm{rpm}$ and at pH 7 are given in table 2. Removal efficiency of different parameter of waste water was achieved at $30 \mathrm{~min}$ contact time from 38 to $94 \%$, i.e. $30 \mathrm{~min}$ contact time was considered because maximum COD removal (64.51\%), BOD (80.0\%), Hexavalent Chromium (85.71\%),Hardness (41.70\%), Nitrite (50.00\%), TSS (50.85\%), TDS (54.28\%). Iron removal was $90.24 \%$ at 30 min contact time. Turbidity was also removed up to $94.15 \%$.

Removal efficiency of different parameter of waste water treated at $30 \mathrm{~min}$ contact time with 5 gm/l GLP dose from 27 to $92 \%$.i.e. $5 \mathrm{gm} / 1$ dosage of GLP was considered as optimum dosage for textile waste water because maximum BOD removal (86.66\%), COD (76.95\%), Hexavalent Chromium (87.14\%), Iron (92.70\%), Turbidity (92.73\%), Hardness (44.64\%), TSS (50.85\%), Nitrite (51.56\%), TDS (65.71\%), Nitrate reduces up to $(27.74 \%)$.

Table 2: Parameter Studied before and after Treatment of Waste Water WITH 10gm/L GLP AT different Contact Time and at pH 7

\begin{tabular}{|c|c|c|c|c|c|c|c|c|}
\hline \multirow{3}{*}{$\begin{array}{l}\text { Sr } \\
\text { no }\end{array}$} & \multirow[t]{3}{*}{ Parameter } & \multirow{3}{*}{$\begin{array}{l}\text { Discharge } \\
\text { Limits of } \\
\text { effluent into } \\
\text { Inland surface } \\
\text { water (mg/l) }\end{array}$} & \multirow{3}{*}{$\begin{array}{l}\text { Initial } \\
(\mathrm{mg} / \mathrm{l})\end{array}$} & \multicolumn{4}{|c|}{ After treatment } & \multirow{3}{*}{$\begin{array}{c}\text { Maximum } \\
\text { \% Removal } \\
\text { (30 min) }\end{array}$} \\
\hline & & & & \multicolumn{4}{|c|}{ Time variable } & \\
\hline & & & & $30 \mathrm{~min}$ & $60 \mathrm{~min}$ & $\begin{array}{c}90 \\
\min \\
\end{array}$ & $120 \mathrm{~min}$ & \\
\hline 1 & $\mathrm{pH}$ & 5.5 to 9.0 & 7 & 7 & 7 & 7 & 7 & - \\
\hline 2 & $\begin{array}{l}\text { Turbidity } \\
\text { NTU }\end{array}$ & - & 217.4 & 12.7 & 13.8 & 14.0 & 14.0 & $94.15 \%$ \\
\hline 3 & COD mg/l & 250 & 3472 & 1232 & 1680 & 1696 & 1728 & $64.51 \%$ \\
\hline 4 & BOD mg/l & 30 & 500 & 100 & 300 & 600 & 900 & $80.0 \%$ \\
\hline 5 & TDS mg/l & 2100 & 3500 & 1600 & 1600 & 1800 & 1700 & $54.28 \%$ \\
\hline 6 & TSS mg/l & 100 & 1628 & 800 & 800 & 900 & 864 & $50.85 \%$ \\
\hline 7 & Hardness mg/l & 600 & 560 & 330 & 340 & 350 & 340 & $41.07 \%$ \\
\hline 8 & Iron $\mathrm{mg} / 1$ & 3 & 2289.8 & 223.4 & 223.4 & 223.4 & 223.4 & $90.24 \%$ \\
\hline 9 & $\begin{array}{l}\text { Hexavalent } \\
\text { Chromium mg/1 }\end{array}$ & 0.1 & 0.70 & 0.10 & 0.11 & 0.10 & 0.10 & $85.71 \%$ \\
\hline 10 & Nitrate $\mathrm{mg} / \mathrm{l}$ & 10 & 31.0 & 19 & 20.1 & 21.0 & 20.8 & $38.70 \%$ \\
\hline 11 & Nitrite $\mathrm{mg} / \mathrm{l}$ & & 0.64 & 0.32 & 0.37 & 0.33 & 0.32 & $50.0 \%$ \\
\hline
\end{tabular}

\section{RESULT OF EFFECT OF GLP DOSE VARIATION ON ADSORPTION OF WASTE WATER CONSTITUENT}

The result of waste water quality parameter at different dosage of GLP at pH 7 and 30 min contact time is given in table 3 . 
Table 3: Parameter Studied before and after Treatment of Waste Water with different GLP dosage 5 gm/l, 10 gm/l, $15 \mathrm{gm} / \mathrm{l}$ AND 20gm/l AND $30 \mathrm{~min}$ Contact time and AT pH 7

\begin{tabular}{|c|c|c|c|c|c|c|c|c|}
\hline \multirow[t]{2}{*}{$\begin{array}{l}\text { Sr } \\
\text { no }\end{array}$} & \multirow[t]{2}{*}{ Parameter } & \multirow{2}{*}{$\begin{array}{l}\text { Dischargeable } \\
\text { Limits of } \\
\text { Inland surface } \\
\text { water (mg/l) }\end{array}$} & \multirow[t]{2}{*}{$\begin{array}{l}\text { Initial } \\
(\mathrm{mg} / \mathrm{l})\end{array}$} & Dose v & ment & & & \multirow[t]{2}{*}{$\begin{array}{l}\text { Maximum } \\
\% \text { Removal } \\
(5 \mathrm{gm} / \mathrm{l})\end{array}$} \\
\hline & & & & $5 \mathrm{gm} / \mathrm{l}$ & $\begin{array}{c}10 \\
\mathrm{gm} / \mathrm{l}\end{array}$ & $\begin{array}{c}15 \\
\mathrm{gm} / \mathrm{l}\end{array}$ & $20 \mathrm{gm} / \mathrm{l}$ & \\
\hline 1 & $\mathrm{pH}$ & 5.5 to 9.0 & 7 & 7 & 7 & 7 & 7 & - \\
\hline 2 & $\begin{array}{l}\text { Turbidity } \\
\text { NTU }\end{array}$ & - & 217.4 & 15.8 & 12.7 & 11.8 & 10.0 & $92.73 \%$ \\
\hline 3 & COD mg/l & 250 & 3472 & 800 & 1232 & 1760 & 1456 & $76.95 \%$ \\
\hline 4 & BOD mg/l & 30 & 500 & 66.67 & 100 & 153.8 & 187.5 & $86.66 \%$ \\
\hline 5 & TDS mg/l & 2100 & 3500 & 1200 & 1600 & 2400 & 2800 & $65.71 \%$ \\
\hline 6 & TSS mg/l & 100 & 1628 & 800 & 800 & 800 & 1200 & $50.85 \%$ \\
\hline 7 & Hardness mg/l & 600 & 560 & 310 & 330 & 340 & 350 & $44.64 \%$ \\
\hline 8 & Iron $\mathrm{mg} / \mathrm{l}$ & 3 & 2289.8 & 167.55 & 223.4 & 446.8 & 614.35 & $92.70 \%$ \\
\hline 9 & $\begin{array}{l}\text { Hexavalent } \\
\text { Chromium mg/l }\end{array}$ & 0.1 & 0.70 & 0.09 & 0.10 & 0.09 & 0.09 & $87.14 \%$ \\
\hline 10 & Nitrate $\mathrm{mg} / \mathrm{l}$ & 10 & 31.0 & 22.4 & 19 & 14.9 & 6.1 & $27.74 \%$ \\
\hline 11 & Nitrite $\mathrm{mg} / \mathrm{l}$ & - & 0.64 & 0.31 & 0.32 & 0.34 & 0.32 & $51.56 \%$ \\
\hline
\end{tabular}

\section{\% REMOVAL EFFICIENCY OF WASTE WATER QUALITY PARAMETER AT DIFFERENT DOSAGE OF GLP AT pH 7 for 30 MIN CONTACT TIME}

Graphical representation for \% removal efficiency of waste water quality parameter at different Dose of GLP is shown in figure $1 . \%$ Removal efficiency of waste water quality parameter at $5 \mathrm{gm} / 1$ GLP dose are Nitrate reduces up to $27.74 \%$, Hardness 44.64\%, TSS 50.85\%, Nitrite 51.56\%, TDS 65.71\%, COD 76.95\%, BOD 86.66\%, Hexavalent Chromium $87.14 \%$, Iron $92.70 \%$, Turbidity $92.73 \%$. 


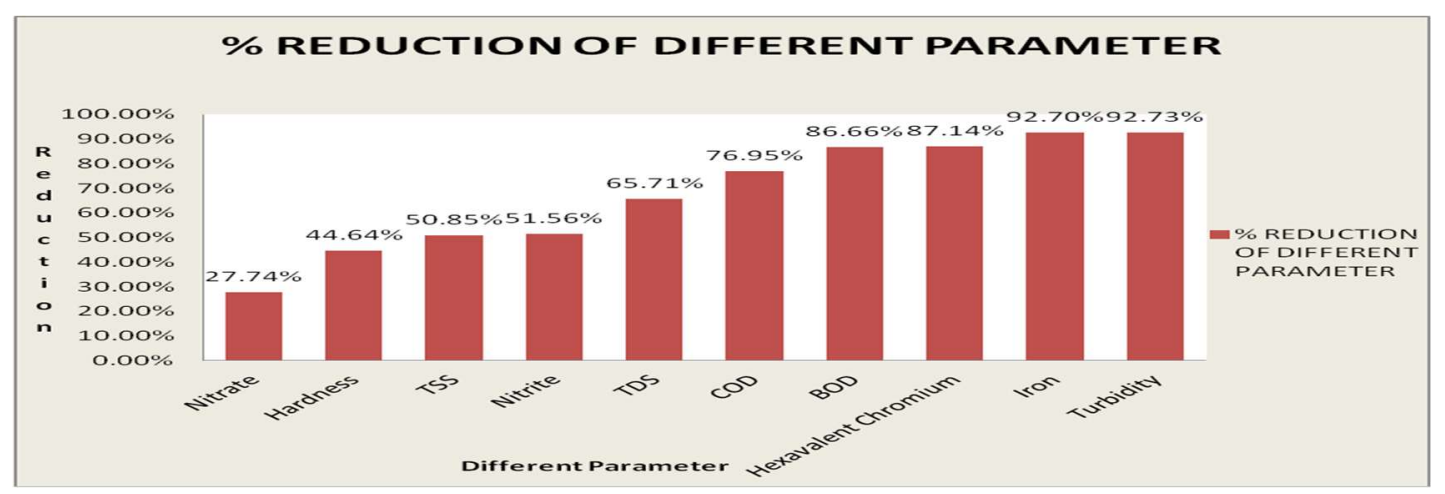

Figure 1: \% Removal efficiency of waste water quality parameter at pH 7 and 30 min contact time and dosage 5 gm/l of GLP.

\section{Adsorption Isotherm:}

Various equilibrium parameters for Langmuir, BET and Freundlich ,Temkin isotherms were calculated [3,4,5,6,8,11,12] and are shown in table 4.

Table 4:Various Equilibrium Parameters For Different Isotherm:

\begin{tabular}{|c|c|c|c|c|c|c|c|c|c|c|c|c|}
\hline \multirow{2}{*}{$\begin{array}{l}\text { Para } \\
\text { meter }\end{array}$} & \multicolumn{3}{|c|}{$\begin{array}{l}\text { Langmuir } \\
\text { Isotherm }\end{array}$} & \multicolumn{3}{|c|}{$\begin{array}{l}\text { Freundlich } \\
\text { Isotherm }\end{array}$} & \multicolumn{3}{|c|}{ Temkin Isotherm } & \multicolumn{3}{|c|}{ BET Isotherm } \\
\hline & $\mathbf{R}^{2}$ & $\begin{array}{l}Q_{0} \\
(\mathrm{mg} / \\
\text { g) }\end{array}$ & $\begin{array}{l}\text { B } \\
(\mathrm{L} / \\
\mathrm{mg})\end{array}$ & $\mathbf{R}^{2}$ & $\begin{array}{l}\text { Kf } \\
(\mathrm{mg} \\
/ \mathrm{g})\end{array}$ & $\begin{array}{l}\mathbf{n} \\
(\mathrm{L} / \\
\mathrm{mg})\end{array}$ & $\mathbf{R 2}$ & $\begin{array}{l}\text { a } \\
\text { (mg } \\
/ g)\end{array}$ & $\begin{array}{l}\mathbf{b} \\
(\mathrm{L} / \\
\mathrm{mg})\end{array}$ & $\mathbf{R 2}$ & $\begin{array}{l}q_{\max } \\
(\mathrm{mg} / \\
\text { g) }\end{array}$ & $\begin{array}{l}\text { B } \\
(\mathrm{L} / \mathrm{m} \\
\text { g) }\end{array}$ \\
\hline $\begin{array}{l}\text { Turbi } \\
\text { dity }\end{array}$ & $\begin{array}{l}0.94 \\
28\end{array}$ & $\begin{array}{l}- \\
9.756 \\
0\end{array}$ & $\begin{array}{l}- \\
0.05 \\
12\end{array}$ & $\begin{array}{l}0.06 \\
09\end{array}$ & $\begin{array}{l}1.44 \\
8\end{array}$ & $\begin{array}{l}1.03 \\
32\end{array}$ & $\begin{array}{l}0.091 \\
86\end{array}$ & $\begin{array}{l}- \\
149 . \\
35\end{array}$ & $\begin{array}{l}67.7 \\
37\end{array}$ & $\begin{array}{l}0.94 \\
02\end{array}$ & $\begin{array}{l}- \\
10.41 \\
66\end{array}$ & -10 \\
\hline COD & $\begin{array}{l}0.88 \\
77\end{array}$ & $\begin{array}{l}62.89 \\
30\end{array}$ & $\begin{array}{l}- \\
0.00 \\
136 \\
8\end{array}$ & $\begin{array}{l}0.90 \\
68\end{array}$ & $\begin{array}{l}100 \\
6 E- \\
10\end{array}$ & $\begin{array}{l}- \\
0.46 \\
15\end{array}$ & $\begin{array}{l}0.922 \\
8\end{array}$ & $\begin{array}{l}435 \\
6.7\end{array}$ & $\begin{array}{l}- \\
576 . \\
15\end{array}$ & $\begin{array}{l}0.91 \\
69\end{array}$ & $\begin{array}{l}40.16 \\
06\end{array}$ & $\begin{array}{l}- \\
3.333 \\
3\end{array}$ \\
\hline BOD & $\begin{array}{l}0.95 \\
36\end{array}$ & $\begin{array}{l}11.06 \\
19\end{array}$ & $\begin{array}{l}- \\
0.01 \\
50\end{array}$ & $\begin{array}{l}0.99 \\
27\end{array}$ & $\begin{array}{l}704 \\
6 E- \\
05\end{array}$ & $\begin{array}{l}- \\
0.62 \\
30\end{array}$ & $\begin{array}{l}0.923 \\
7\end{array}$ & $\begin{array}{l}358 . \\
26\end{array}$ & $\begin{array}{l}- \\
66.4 \\
6\end{array}$ & $\begin{array}{l}0.92 \\
88\end{array}$ & $\begin{array}{l}7.961 \\
7\end{array}$ & $\begin{array}{l}- \\
6.043 \\
2\end{array}$ \\
\hline TDS & $\begin{array}{l}0.86 \\
22\end{array}$ & $\begin{array}{l}22.32 \\
14\end{array}$ & $\begin{array}{l}- \\
0.00 \\
076 \\
4\end{array}$ & $\begin{array}{l}0.98 \\
76\end{array}$ & $\begin{array}{l}370 \\
7 E- \\
12\end{array}$ & $\begin{array}{l}- \\
0.34 \\
56\end{array}$ & $\begin{array}{l}0.907 \\
6\end{array}$ & $\begin{array}{l}377 \\
0.6\end{array}$ & $\begin{array}{l}- \\
474 . \\
48\end{array}$ & $\begin{array}{l}0.75 \\
63\end{array}$ & $\begin{array}{l}7.593 \\
0\end{array}$ & $\begin{array}{l}- \\
1.490 \\
9\end{array}$ \\
\hline TSS & 0.97 & 8.620 & - & 0.71 & 215 & - & 0.419 & 141 & - & 0.99 & 3.866 & - \\
\hline
\end{tabular}




\begin{tabular}{|c|c|c|c|c|c|c|c|c|c|c|c|c|}
\hline & 19 & 6 & $\begin{array}{l}0.00 \\
139 \\
4\end{array}$ & 84 & $\begin{array}{l}8 E- \\
13\end{array}$ & $\begin{array}{l}0.27 \\
98\end{array}$ & 8 & 6.8 & $\begin{array}{l}196 . \\
81\end{array}$ & 37 & 9 & $\begin{array}{l}1.139 \\
6\end{array}$ \\
\hline $\begin{array}{l}\text { Hardn } \\
\text { ess }\end{array}$ & $\begin{array}{l}0.94 \\
8\end{array}$ & $\begin{array}{l}1.503 \\
7\end{array}$ & $\begin{array}{l}- \\
0.00 \\
329 \\
4\end{array}$ & $\begin{array}{l}0.99 \\
85\end{array}$ & $\begin{array}{l}732 \\
8 E- \\
35\end{array}$ & $\begin{array}{l}- \\
0.07 \\
51\end{array}$ & $\begin{array}{l}0.962 \\
2\end{array}$ & $\begin{array}{l}197 \\
1.1\end{array}$ & $\begin{array}{l}- \\
335 . \\
3\end{array}$ & $\begin{array}{l}0.93 \\
53\end{array}$ & $\begin{array}{l}1.204 \\
9\end{array}$ & $\begin{array}{l}- \\
0.832 \\
5\end{array}$ \\
\hline Iron & $\begin{array}{l}0.97 \\
33\end{array}$ & $\begin{array}{l}66.66 \\
67\end{array}$ & $\begin{array}{l}- \\
0.00 \\
641 \\
7\end{array}$ & $\begin{array}{l}0.94 \\
52\end{array}$ & $\begin{array}{l}120 \\
7 E- \\
06\end{array}$ & $\begin{array}{l}- \\
0.88 \\
05\end{array}$ & $\begin{array}{l}0.822 \\
1\end{array}$ & $\begin{array}{l}153 \\
2.5\end{array}$ & $\begin{array}{l}- \\
229 . \\
57\end{array}$ & $\begin{array}{l}0.69 \\
6\end{array}$ & $\begin{array}{l}68.49 \\
31\end{array}$ & -73 \\
\hline $\begin{array}{l}\text { Hexav } \\
\text { alent } \\
\text { Chro } \\
\text { mium }\end{array}$ & $\begin{array}{l}0.02 \\
6\end{array}$ & $\begin{array}{l}0.033 \\
2\end{array}$ & $\begin{array}{l}- \\
6.43 \\
29\end{array}$ & $\begin{array}{l}0.95 \\
66\end{array}$ & $\begin{array}{l}5.84 \\
3\end{array}$ & $\begin{array}{l}- \\
5.06 \\
32\end{array}$ & $\begin{array}{l}0.002 \\
8\end{array}$ & $\begin{array}{l}- \\
0.03 \\
54\end{array}$ & $\begin{array}{l}- \\
0.04 \\
14\end{array}$ & $\begin{array}{l}0.02 \\
15\end{array}$ & $\begin{array}{l}- \\
0.041 \\
5\end{array}$ & $\begin{array}{l}- \\
3.317 \\
8\end{array}$ \\
\hline $\begin{array}{l}\text { Nitrat } \\
\text { e }\end{array}$ & $\begin{array}{l}0.71 \\
66\end{array}$ & $\begin{array}{l}1.724 \\
7\end{array}$ & $\begin{array}{l}0.20 \\
23\end{array}$ & $\begin{array}{l}0.13 \\
85\end{array}$ & $\begin{array}{l}1.09 \\
4\end{array}$ & $\begin{array}{l}7.68 \\
63\end{array}$ & $\begin{array}{l}0.162 \\
2\end{array}$ & 0.79 & $\begin{array}{l}0.19 \\
67\end{array}$ & $\begin{array}{l}0.99 \\
27\end{array}$ & $\begin{array}{l}0.389 \\
2\end{array}$ & $\begin{array}{l}- \\
8.038 \\
7\end{array}$ \\
\hline Nitrite & $\begin{array}{l}0.39 \\
52\end{array}$ & $\begin{array}{l}0.002 \\
90\end{array}$ & $\begin{array}{l}- \\
3.50 \\
85\end{array}$ & $\begin{array}{l}0.44 \\
3\end{array}$ & $\begin{array}{l}708 \\
9 E- \\
07\end{array}$ & $\begin{array}{l}- \\
0.09 \\
26\end{array}$ & $\begin{array}{l}0.494 \\
5\end{array}$ & $\begin{array}{l}- \\
0.43 \\
46\end{array}$ & $\begin{array}{l}- \\
0.41 \\
35\end{array}$ & $\begin{array}{l}0.46 \\
52\end{array}$ & $\begin{array}{l}0.002 \\
33\end{array}$ & $\begin{array}{l}- \\
1.216 \\
4\end{array}$ \\
\hline
\end{tabular}

Experimental data obtained by above analysis were tested with adsorption models....The values of coefficient of correlation $\left(\mathrm{R}^{2}\right)$ in good agreement with Langmuir, Freundlich, BET, Temkin isotherms. Values of coefficient of correlation $\left(R^{2}\right)$ are not nearer to 1.0. Therefore, it is confirmed that the adsorption process occurred over here is quite good process.[2,4]Some of the best fits are represented here for adsorption isotherms in figure 6.Langmuir isotherms suggests homogenous surface by monolayer adsorption. BET isotherms indicate monolayer sorption and subsequent layers.[13] Temkin and Freundlich isotherms shows heterogeneous surface by multilayer adsorption. The Temkin isotherm fits the present data because it takes into account the occupation of the more energetic adsorption sites at first.[3]

Experimental data obtained by above analysis were tested with adsorption models....The best fit for Turbidity is obtained for Temkin isotherm. The GLP also followed Temkin isotherm as the value of $b$ is 67.737 in Turbidity. The Temkin isotherm fits the present data because it takes into account the occupation of the more energetic adsorption sites at first. The best fit for COD, BOD, TDS, TSS and Hardness is obtained for Langmuir and BET isotherm. The best fit for Iron is obtained for Langmuir isotherm. Chromium Hexavalent dose not the followed any adsorption isotherm. Chromium hexavalent followed the Freundlich isotherm in reverse manner i.e. with increasing dosage qe decreasing. The best fit for Nitrate is obtained for BET isotherm. Nitrite followed the adsorption pattern of Langmuir and BET isotherm. 
Some of the Best fit Adsorption Isotherms obtained in the various models are shown here in figure 2.
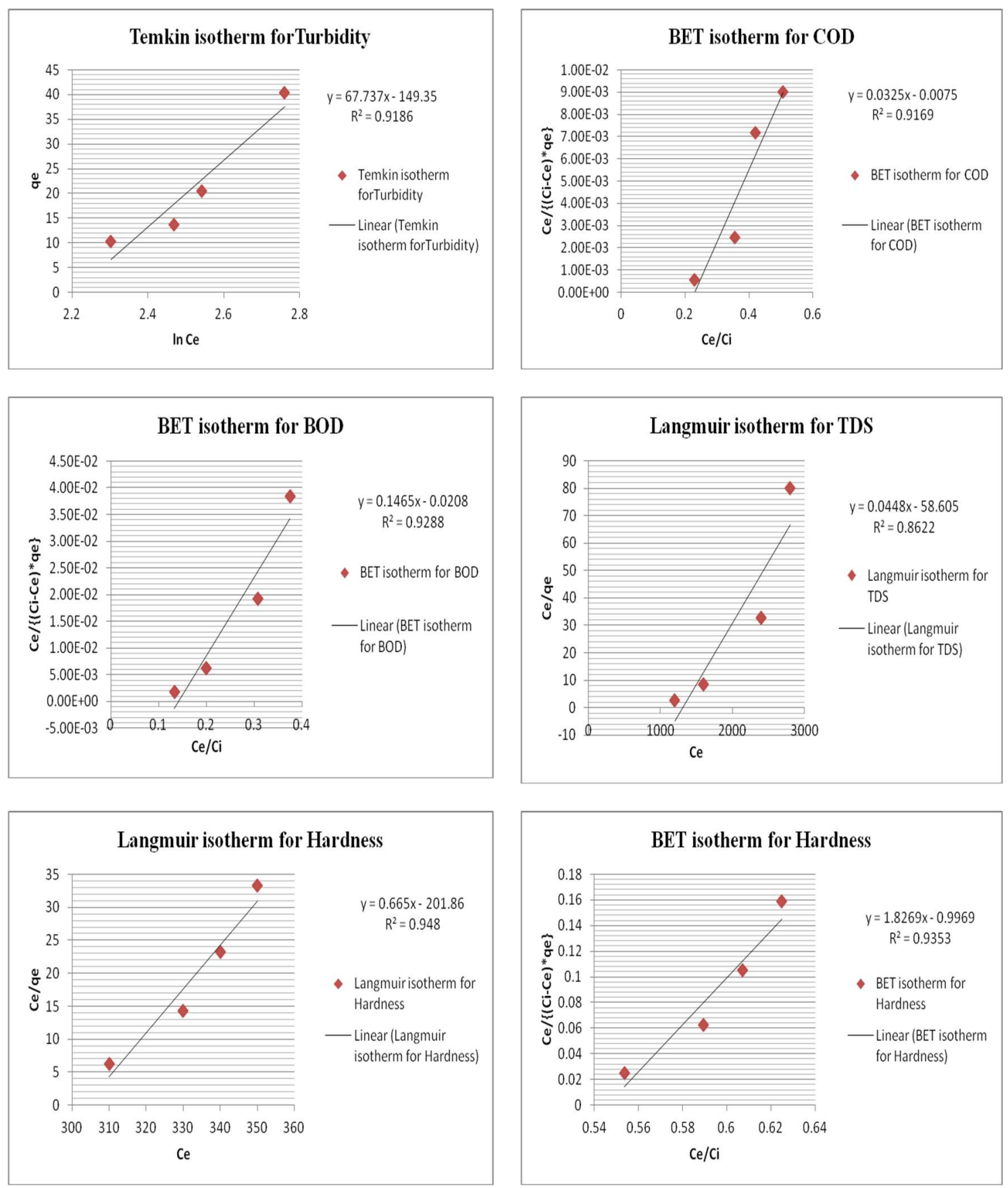

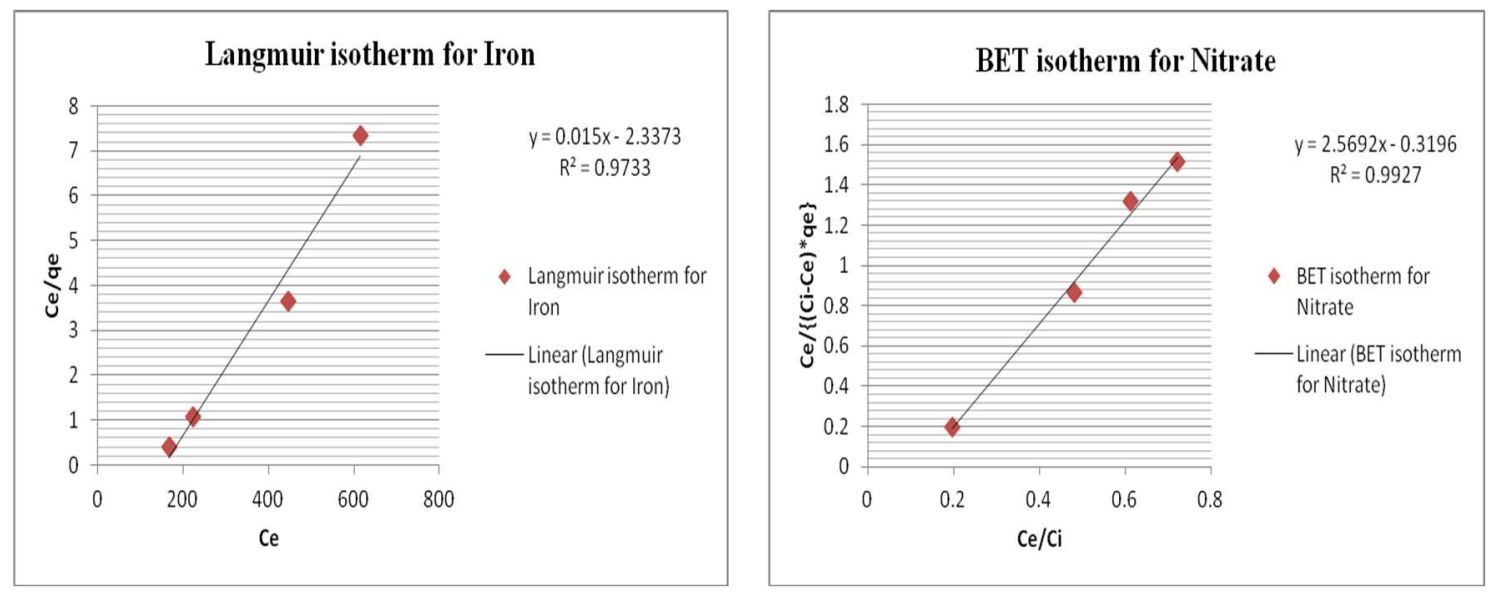

Figure 2: Best fit Adsorption Isotherm obtained in the various models.

The FTIR report of GLP shows the different functional group are present in it is shown in figure 3 . The FTIR report of GLP shows the presence of different functional groups $(-\mathrm{OH}$, alkanol, phenol, $-\mathrm{C}=\mathrm{O}$ Carbonyl group, Aldehyde, ester, $-\mathrm{C}=\mathrm{C}$ - alkene, $-\mathrm{CH}_{3}$ bend, $\mathrm{C}-\mathrm{O}, \mathrm{COOH},-\mathrm{O}-, \mathrm{COOR},-\mathrm{CN}$ amines $)$ in it. The experimental values are compared with standard value[5,9] is shown in table 5 .

The samples were treated with GLP adsorbent-natural electrolyte by directly applying the different dosage of adsorbent. It increases the nutrition values of the treated water. Under adequate agitation these particles then grow in size to form flocks, which can be settled by gravity or be removed by filtration any matter present in the suspended form than they can adsorb on the surface of adsorbent.

\begin{tabular}{|c|c|c|}
\hline & & Table 5: Identification of Peaks of FTIR \\
\hline Wave numl & $\mathrm{v} \mathrm{cm}^{-1}$ & Types of Vibration \\
\hline Standard & $\begin{array}{l}\text { Experim } \\
\text { ental }\end{array}$ & \\
\hline $3500-3200$ & $\begin{array}{l}3363.00 \\
3292.60\end{array}$ & $\begin{array}{l}\text {-OH, alkol, phenol, H-Bond free } \\
\text { (isolated) }\end{array}$ \\
\hline $3150-3050$ & $\begin{array}{l}3181.69 \\
3064.99\end{array}$ & Aromatic stretch \\
\hline $2850-2990$ & 2974.33 & -CH, alkane \\
\hline $2900-2700$ & $\begin{array}{l}2749.62 \\
2656.07\end{array}$ & Aldehyde stretch \\
\hline $1740-1710$ & 1734.06 & $\begin{array}{l}-\mathrm{C}=\mathrm{O} \text { Carbonyl group, } \\
\text { Aldehyde, ester }\end{array}$ \\
\hline $1680-1600$ & 1653.05 & $-\mathrm{C}=\mathrm{C}-$ alkene \\
\hline
\end{tabular}




\begin{tabular}{|c|c|c|}
\hline $1600-1480$ & $\begin{array}{l}1556.61 \\
1512.24\end{array}$ & Aromatic \\
\hline $1450-1390$ & $\begin{array}{l}1452.45 \\
1370.47\end{array}$ & $-\mathrm{CH}_{3}$ bend \\
\hline $1000-1350$ & $\begin{array}{l}1319.35 \\
1245.09 \\
1141.90 \\
1102.35 \\
1060.88 \\
1027.13\end{array}$ & $\begin{array}{l}\mathrm{C}-\mathrm{O}, \mathrm{OH}, \mathrm{COOH},-\mathrm{O}-\text {, } \\
\mathrm{COOR} \\
\text {-CN amines }\end{array}$ \\
\hline $900-650$ & $\begin{array}{l}1002.05 \\
828.45 \\
668.36\end{array}$ & Out of plane bend \\
\hline
\end{tabular}

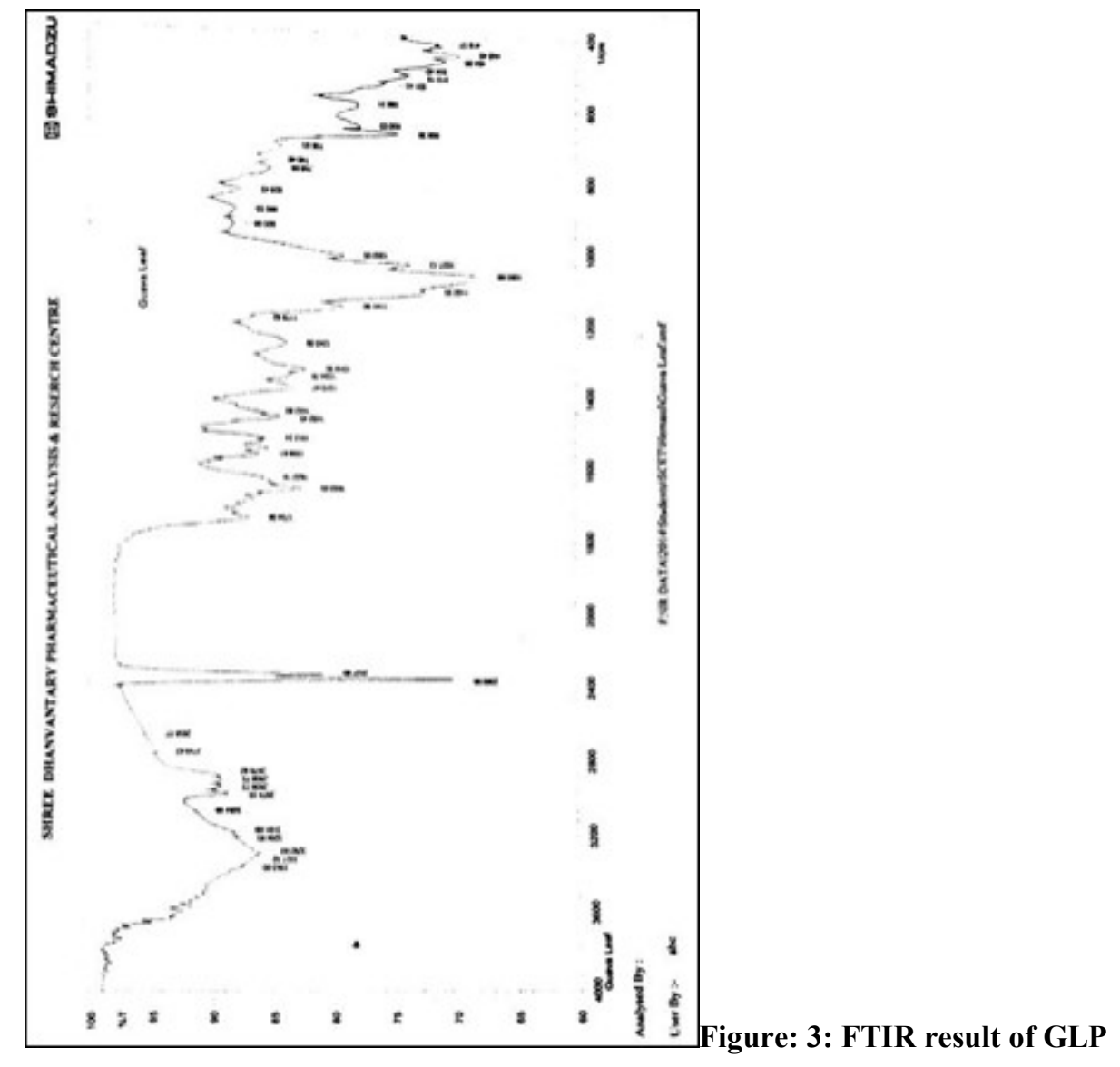




\section{CONCLUSIONS}

Any Wastewater can be treated with GLP (5 gm/l) dosage , 30 min contact time and at $7 \mathrm{pH}$. After treating with this cost effective, eco- friendly technique, the water quality parameters are found within the range of drinking water quality. This technique will save cost of chemicals and operation-maintenance of treatment plant and will not produce hazardous sludge. We can directly apply. No primary treatment is required before applying adsorbent to the sample. It is a cheap and easy method for treatment of effluent. This method is efficient because GLP used is biodegradable and can be used as a part in fertilizing materials for agriculture purpose.

\section{REFERENCES}

1. Patel Kamal, Desai Hitesh and Desai Hemangi, Impact Of Industrialization And Urbanization On Water Quality Of River Tapi (Surat, Gujarat, India), International Journal of Environmental Research and Development, 9(2),306-317,Oct-Dec 2014.

2. Karale, Sheetal S., and MAYUR M. Suryavanshi. "Dairy wastewater treatment using coconut shell activated carbon \& laterite as low cost adsorbents." Int. J. Civil, Struct. Environ. Infrastruct. Eng. Res. Dev 1 (2014): 9-14.

3. Patel Niyati and Desai Hemangi, Potential of Moringa Oleifera seeds, Leaves and Bark for removal of Hexavalent Chromium from Aqueous Solution with reference to Adsorption Isotherm, International Journal of Water Resources \& Environmental Management, 2(1), 41-57, Jan-June 2011.

4. Andrew D.Eaton, Lenore S. Clesceri, Eugene W. Rice Arnold E. Greenberg, Standard Methods for the Examination of Water

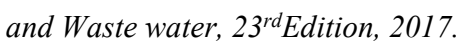

5. Rani, K., et al. "Biological Treatment Of Distillery Waste Water-An Overviewll." International Journal of General Engineering and Technology 2.4 (2013): 15-24.

6. Raval Priti and Desai Hemangi, Removal of anionic surfactant sodium dodecyl sulphate (SDS) from aqueous solution by using Alumina, International Journal of Environmental Research and Development, 7 (2A), 851-862, Oct-Dec 2012.

7. Desai Birva and Desai Hemangi, Potential of Moringa Oleifera(Drum Sticks) Seeds and its Application as Natural Adsorbent in removal of Heavy Metal ions from Water, International Journal of Environment, Ecology, Family and Urban Studies, 3(4),9-22, October 2013.

8. Desai Akruti and Desai Hemangi Potential ofAzadirachta indicaLeaves for Removal of $\mathrm{Cr}^{+6}$ from Aqueous Solution with reference to Adsorption Isotherm, International Journal of Innovative Research and Development, 3(7), 359-368, July 2014.

9. Adhoni, Shakeel Ahmed, Shanthanu M. Raikar, and C. T. Shivasharana. "Bioremediation of Industrial Effluents with Heavy Metals using Immobilised Microalgae." International Journal of Applied and Natural Sciences (IJANS) 7.5 (2018): 67-84.

10. Raval Priti and Desai Hemangi, Potential of cationic surfactant modified silica gel in removal of organophosphate pesticideMonocrotophos from aqueous solution, International Journal of Environment, Ecology, Family and Urban Studies, 6(1), 2538, February 2016.

11. Gamit Payal and Desai Hemangi, A Study On The Effect Of Landfill Leachate-Pollution On Ground Water And Surface Water Quality And Leachate Treatment With Activated Carbon Adsorbent, International Journal of Environmental Research And Development, 11(3), 471-481, January-March 2017.

12. Desai Hitesh and Desai Hemangi, A Green Water Technology: Groundwater Quality Investigation - Treatment with Natural Polyelectrolyte, International Journal of Applied Environmental Sciences, 12( 5), 755-772,2017. 
13. Gnanavel, G., and P. Muthusamy. "PHARMACEUTICAL INDUSTRY WASTEWATER TREATMENT USING ATMOSPHERIC AIR AND PURE OXYGEN." IASET: International Journal of Metallurgical, Materials and Chemical Engineering (IASET: IJMMCE) 7.6, Oct - Nov 2018; 1-6

14. Desai Birva and Desai Hemangi ,Evaluation of Water Quality Index for Ground Water of Residential Area of Surat City, Gujarat, India, SSRG International Journal of Agriculture \& Environmental Sciences, 5(3), 53-65, May-June 2018.

15. Raval Priti and Desai Hemangi, Potential Of Anionic Surfactant Modified Alumina in Removal of Crystal Violet from Aqueous Solution, International Journal of Research and Analytical Reviews, 6(1), 145-158, March 2019.

16. Diyora Krupali and Desai Hemangi, The castor seed husk used as an effective natural adsorbent for treatment of textile effluent,Journal of Environmental Research and Development, 13 (3),214-225, January-March 2019.

17. 13. Krupa Lakhani and Desai Hemangi, The millet husk used as effective natural adsorbent for removal of cod and water pollutants, International Journal of Research and Analytical Reviews, 6(2),123-135,April-June 2019. 